\title{
ARTICLE
}

\section{Development of high-performance gel-type radiation shielding material using polymer resin}

\author{
Naoteru Odano*, Akiko Konnai and Mitsufumi Asami \\ National Maritime Research Institute,6-38-1 Shinkawa, Mitaka, Tokyo 181-0004, Japan
}

\begin{abstract}
New gel-type shielding material, mainly consists of resin, lead powder and a boron compound, was developed. For simplification of the production process, new production method blending two types of liquid of base resin and a hardening accelerator was developed. To produce base resin, the crosslinking agent and the polymerization agent were blended with the thermosetting resin, first, and boric acid and lead were blended with the oligomerized component. Although the blended material is in gel condition immediately after blending, it has the feature hardened automatically without any processing. Radiation shielding performance of developed gel-type shielding material for neutron and gamma ray up to $25 \mathrm{~cm}$ in thickness were measured using standard sources of ${ }^{252} \mathrm{Cf}$ and ${ }^{60} \mathrm{Co}$. It was found that new shielding material was able to reduce neutron dose rate half for that of ordinary concrete and shielding performance for gamma-ray was slightly good compared with that of ordinary concrete. Monte Carlo simulations were also carried out to confirm radiation shielding performance of newly developed gel-type shielding material for neutron and gamma ray up to 100 $\mathrm{cm}$ in thickness. From the experiments and simulations, it was shown that newly developed gel-type shielding material has excellent shielding performance compared with that of ordinary concrete, and can be used for compensated shield in the case of accident.
\end{abstract}

Keywords: shielding material; resin; polymer; gel-type; neutron; gamma-ray; secondary gamma-ray; Monte Carlo

\section{Introduction}

As the utilization of nuclear energy is diversified, the neutron shielding materials with heat resistance, flexibility, and low activation concrete have been developed [1-3].

National Maritime Research Institute (NMRI) has also been conducting research and development of high performance radiation shielding material [4]. The developed shielding material has shield effectiveness for both the neutron and gamma-ray and the shielding performance of the developed material was the largest in comparison with a practical shielding material. Moreover, a property of low activation had demonstrated by the neutron activation experiment.

Principal properties of previously developed high performance radiation shielding material developed by NMRI are as follows [4]; (1) the developed material consists of stearyl methacrylate as a high-hydrogen concentrate material, lead as heavy metal, and boric acid as neutron absorber, (2) density was $2.35 \mathrm{~g} / \mathrm{cm}^{3}$, (3) 46 $\mathrm{cm}$ in thickness of newly developed material has equivalent shielding ability to $100 \mathrm{~cm}$ in thickness of ordinary concrete.

While the production method of the previously

*Corresponding author. Email: odano@nmri.go.jp developed shielding material is heat curing that compound of resin, lead powder and boric acid are heated to harden, the process of curing is complex and it was difficult to produce thick shielding material due to limitation of size of heating equipment. To improve the problem in curing of the material, new method easily to cure the gel-type shielding material for nuclear power plant, nuclear fuel cycle facilities, and transport casks base on composition previously developed.

In this paper, newly developed method to produce shielding material using polymer resin and results of evaluation of shielding performance are presented.

\section{Development of gel-type radiation shielding material}

\subsection{Required properties of gel-type radiation shielding material}

The present research is aimed at developing high-performance shielding material that can be used as the improvised one to compensate for the damage of shields due to accident during operation of nuclear power plants, nuclear fuel cycle facilities and transport casks. Goals of the shielding performance are as follows, (1) maintain excellent shielding performance 
for both neutron and gamma-ray as found in the heat curing shielding material, (2) maintain density of the developed material same as ordinary concrete.

For composition of the material, resin as high hydrogen concentrate material, lead as heavy metal and boron as neutron absorber were used same as the heat curing shielding material developed by NMRI [4].

Since the heat curing shielding material needed to prepare complex process and curing equipment for production of shielding material, in this study, we aimed at developing the production method with natural hardening process which can be used for an emergency response. For this purpose, a production method to blend two types of liquid of base resin and a hardening accelerator has been adopted for immediate use in emergency response by preparing two types of liquid as one of materials for emergency preparedness. Considering utilization as compensated shielding materials, it is important to maintain the adhesive strength between target materials and additional compensated shield.

\subsection{Procedure for production of radiation shielding material}

\subsubsection{Outline of Procedure for production of radiation} shielding material

As shown in Figure 1, the radiation shielding material was produced by blending two types of liquid. Two liquids are as follows; one is basic material composing shielding material such as polymer resin, boric acid and lead, and another is hardening accelerator. The atomized powder of lead was used for heavy metal.

Since thermosetting resin has generally small viscosity, homogeneity of produced shielding material has been maintained by adding process of oligomerization. The process that polymerized material is added to monomer and heated enables us to advance the dispersion of boric acid and lead.

As the precursor stage of polymerization, it is necessary to produce oligomer, polymer bind to relatively small quantity of monomer. The shielding material developed in this study is stored with two types of liquid, in order to construct immediately in case of accident, which boric acid and lead are added to oligomer and stored in liquid.

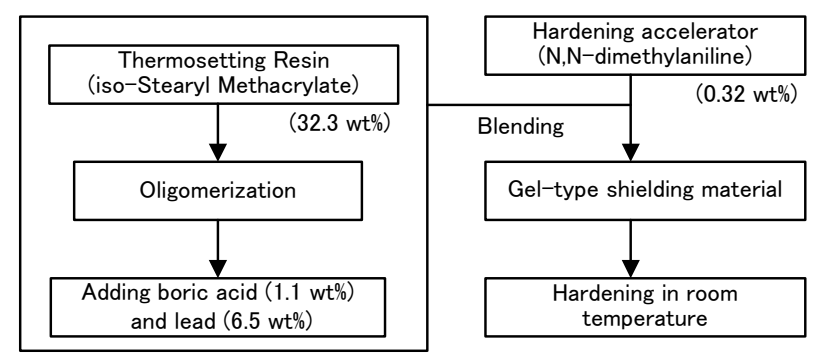

Figure 1. Schematic outline of Procedure for production of radiation shielding material. The weight ratios of raw materials are shown in parentheses.

\subsubsection{Selection of monomer and hardening accelerator}

Appropriate selection of polymer resin and hardening accelerator is important to achieve goals that establishing production methods of shielding material by blending two types of liquid, shielding performance especially for neutron, and the adhesive property to target material which compensated shield would be installed.

There are two criteria to select a suitable monomer. The monomer should be available as adhesive and radical polymerizable. Moreover, the monomer should be easily controlled in hardening reaction and contain many hydrogen atoms as possible. Iso-stearyl methacrylate (isoSMA) was selected as a suitable monomer based on the above mentioned criteria. Since the isoSMA contains iso-stearyl group which contains branch structures, the produced oligomer from isoSMA is less subject to hardening, compared with SMA (Stearyl Methacrylate), which contains stearyl group whose number of carbon atoms are same as that of iso-stearyl group. This makes possible the procedure for producing shielding material by blending two types of liquid when constructing compensated shield in emergency situation. Since isoSMA has the number density of hydrogen atoms which is double compared with epoxy resin, the latter criteria can also be satisfied.

Appropriate selection of hardening accelerator is important from the view point of hardening process at room temperature. Considering the reaction to polymerizable monomer, the N,N-dimethylaniline (DMA), which would be expected hardening at room temperature was selected.

\subsubsection{Stability of liquid raw material during storage}

To allow blending the two types of liquid when constructing, stability of long-term storage is required. A liquid with oligomer adding boric acid and lead powder, and DMA were stored for 151 days at $5{ }^{\circ} \mathrm{C}$ to verify the stability for long term storage. It was found that the two types of liquid stored for about half a year could be hardened within 24 hours after blending. The present result shows it could be possible to store these material for a long term and available as shielding material after storage.

\subsection{Production of gel-type radiation shielding material}

Slab-type shielding materials were produced by means of the procedure described in sections 2.2.1 and 2.2.2. Atomic composition of produced shielding material obtained from weight of raw materials is shown in Table 1. The dimension of the produced shielding material was $31 \mathrm{~cm}$ square and $5 \mathrm{~cm}$ in thickness. The measured densities of the produced materials ranged

Table 1. Atomic composition of produced shielding material.

\begin{tabular}{ccccccc}
\hline \multicolumn{7}{c}{ Atomic composition (weight \%) } \\
$\mathrm{C}$ & $\mathrm{H}$ & $\mathrm{O}$ & $\mathrm{N}$ & $\mathrm{Pb}$ & $\mathrm{B}$ & Total \\
\hline 26.28 & 4.20 & 4.24 & 0.04 & 65.05 & 0.20 & 100 \\
\hline
\end{tabular}


from 2.3 to $2.4 \mathrm{~g} / \mathrm{cm}^{3}$.

\section{Shielding performance of developed material}

\subsection{Experiments to confirm radiation shielding performance for neutron and gamma-ray}

Shielding experiments were carried out to confirm the shielding performance of the developed material using a ${ }^{252} \mathrm{Cf}$ neutron source and a ${ }^{60} \mathrm{Co}$ gamma-ray source. Shielding experiments were carried out for slab arrangement. Thickness of shielding material was increased by $5 \mathrm{~cm}$ up to $25 \mathrm{~cm}$. Radiation shielding performance was evaluated by measurement of dose rate using rem counter for neutron and NaI scintillation counter for gamma ray, respectively. The distance from the source position to the detector was fixed to $146 \mathrm{~cm}$ for neutron measurement and $56 \mathrm{~cm}$ for gamma-ray measurement, respectively.

The results of shielding experiment are shown in Figure 2. While the density of the developed shielding material was 2.3 to $2.4 \mathrm{~g} / \mathrm{cm}^{3}$ and slightly higher than that of ordinary concrete, as shown in Figure 2, the developed shielding material showed excellent shielding performance for neutron and gamma ray compared with ordinary concrete. Comparing neutron dose rate reduction by same thickness of $25 \mathrm{~cm}$ shielding material, it was found that the shielding ability of developed material was approximately twice compared with that of ordinary concrete. This excellent shielding ability is mainly attributed to high number density of hydrogen atom compared with the ordinary concrete and added boron as neutron absorber.

Due to the limitation of size of shielding material, room scattering was not negligible in this experiment. To demonstrate shielding performance of the developed shielding material for deep penetration, Monte Carlo simulations were carried. The details of the simulations and the results are discussed in Section 3.2.

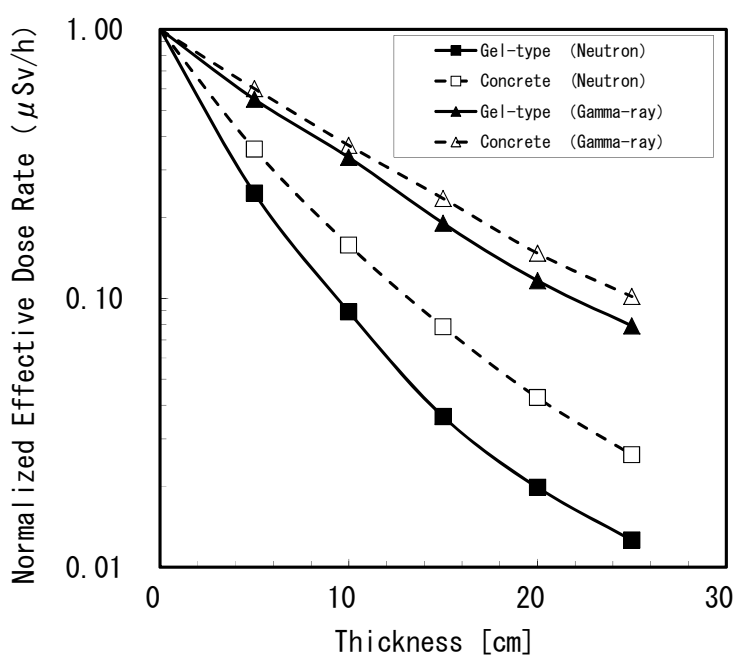

Figure 2. Experimental results of shielding performance of developed shielding material for neutron and gamma-ray compared with ordinary concrete.

\subsection{Evaluation of shielding performance by Monte Carlo simulation}

Monte Carlo simulations were carried out to confirm shielding performance of developed materials for neutron, secondary gamma ray induced by neutron, and gamma ray up to $100 \mathrm{~cm}$. The MCNP5 code [5] with neutron nuclear data library based on JENDL-3.3 [6] and photoatomic data library MCPLIB04 based on data from ENDF/B-VI release 8 [7] was used for the simulations. One dimensional slab arrangement of shielding material was adopted for the calculation model.

\subsubsection{Neutron shielding performance}

Evaluated shielding performances for neutrons from ${ }^{252} \mathrm{Cf}$ up to $100 \mathrm{~cm}$ thickness are shown in Figure 3. The developed shielding material has excellent shielding performance for neutron compared with ordinary concrete. Necessary shielding thickness of the developed material to reduce effective dose comparative with 100 $\mathrm{cm}$ of ordinary concrete was $42 \mathrm{~cm}$. This shows that the thickness less than half is enough for the developed shielding material to reduce dose rate in same level by using the ordinary concrete.

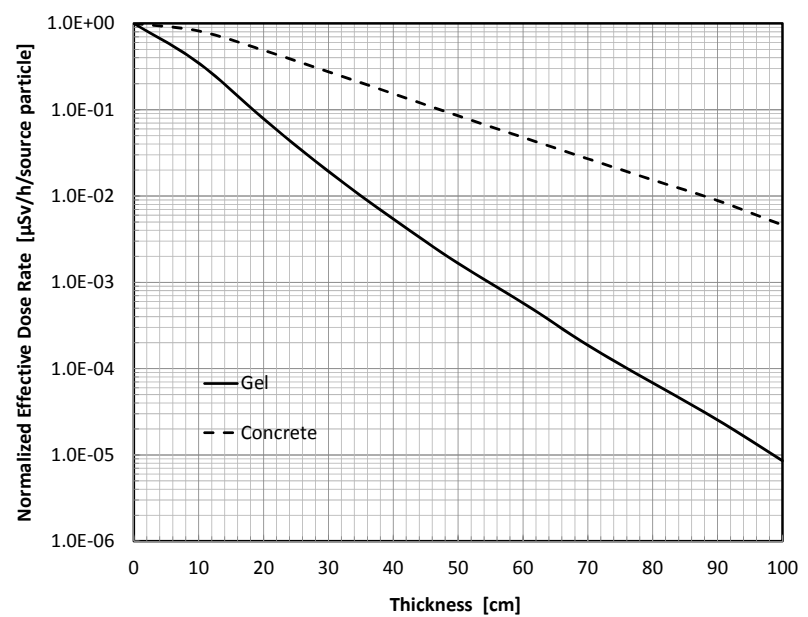

Figure 3. Results of Monte Carlo calculation for evaluation of shielding performance of developed shielding material (Gel) and ordinary concrete for neutron.

\subsubsection{Secondary gamma-ray shielding performance}

Evaluated shielding performances for secondary gamma ray induced by neutrons from ${ }^{252} \mathrm{Cf}$ up to $100 \mathrm{~cm}$ thickness are shown in Figure 4. Since the developed shielding material contains 0.2 weight \% of boron, secondary gamma-ray induced by thermal neutron could be suppressed. Therefore significant effect of suppression of the secondary gamma ray can be found more than $50 \mathrm{~cm}$ in thickness of the developed material. It was also found that level of production of secondary gamma-ray by the developed shielding material at 100 $\mathrm{cm}$ was almost same level as lead.

\subsubsection{Gamma-ray shielding performance}

Evaluated shielding performances for gamma rays 
from ${ }^{60} \mathrm{Co}$ up to $100 \mathrm{~cm}$ thickness are shown in Figure 5. The shielding performance for the developed shielding material was slightly better than that of ordinary concrete. The present results show that approximately $80 \%$ of the thickness enough for the developed shielding material to reduce dose rate in same level by using the ordinary concrete.

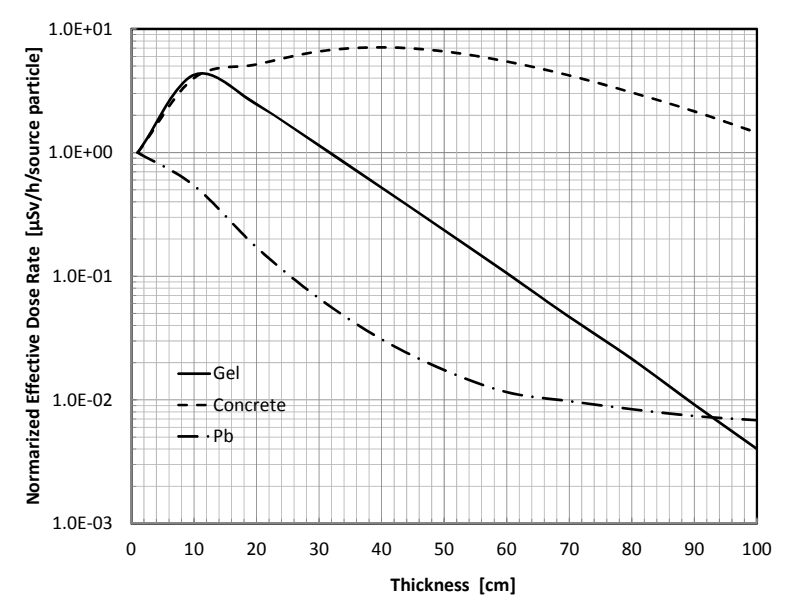

Figure 4. Results of Monte Carlo calculations for evaluation of shielding performance of developed shielding material (Gel), ordinary concrete and lead for neutron induced secondary gamma-ray.

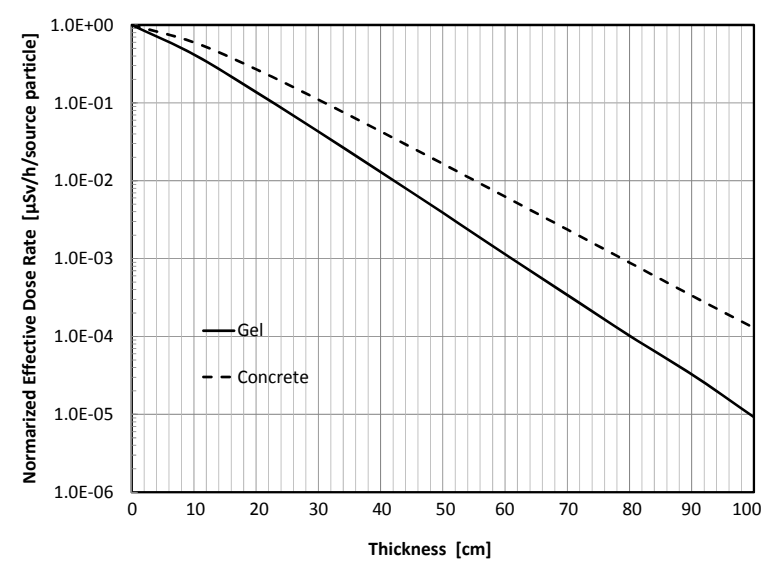

Figure 5. Results of Monte Carlo calculations for evaluation of shielding performance of developed shielding material (Gel) and ordinary concrete for ${ }^{60} \mathrm{Co}$ gamma-ray.

\section{Conclusion}

In this study, new gel-type shielding material for emergency condition for nuclear power plant, nuclear fuel cycle facility, and transport cask was developed and its shielding performances were evaluated.

New production method blending two types of liquid, one is isoSMA oligomer adding boric acid and lead, another one is hardening accelerator, at the time of shielding construction was developed. It was demonstrated that shielding material could be hardened at room temperature without any hardening process. This method would make possible ease construction of material for compensating for lack of radiation shield at emergency condition. The developed shielding material is also applicable to compensated shields for the complex part of shielding structure such as penetration due to its flowability.

Shielding experiments were carried out to evaluate shielding performance for neutron and gamma ray. Experimental results indicated that the developed shielding material has excellent shielding performance especially for neutron compared with ordinary concrete. The Monte Carlo simulations were also indicated that shield effect for the secondary gamma ray was significantly excellent, because boron was added to the shielding material as neutron absorber. These results shows that the developed shielding material could contribute to the weight reduction of neutron shielding and reduction of radioactive waste occurred by decommissioning of nuclear facilities.

\section{Acknowledgements}

This study was supported by the Budget for Nuclear Research of the Ministry of Education, Culture, Sports, Science and Technology.

\section{References}

[1] A. Morioka, S. Sakurai, K. Okuno and H. Tamai, Development of a heat-resistant neutron shielding resin for the national centralized Tokamak, J. Plasma Fusion Res. 81 (2005), pp. 645-646.

[2] A.M. Sukegawa. Y. Anayama, S. Ohnishi, S. Sakurai, A. Kaminaga and K. Okuno, Development of flexible neutron-shielding resin as an additional shielding material, J. Nucl. Sci.Technol. 48 (2011), pp. 585-590.

[3] A. Hasegawa, M. Kinno, M. Uematsu, K. Hayashi, M. Nakata, T. Tanosaki, R. Yoshino and M. Sato, Low-activation reinforced concrete design methodology (1) - Overview of the project, SMiRT-19 (2007).

[4] T. Miura, Y. Hirao, T. Hayashi, K. Okuno and T. Ishida, Study on the Development of Shielding Materials Using Resin, Heavy Material and Thermal Neutron Absorber as Raw Materials, Report of National Maritime Research Institute, 2 (2002), pp. 411-447. [in Japanese]

[5] X-5 Monte Carlo Team, Los Alamos National Laboratory (LANL), MCNP-A General Monte Carlo N-Particle Transport Code, Version 5, LA-UR-03-1987 (2003).

[6] K. Shibata, T. Kawano, T. Nakagawa et al., Japanese evaluated nuclear data library Version 3 Revision-3: JENDL-3.3, J. Nucl. Sci. Technol. 39 (2002), pp. 1125-1136.

[7] M.C. White, Photoatomic Data Library MCPLIB04: A New Photoatomic Library Based on Data from ENDF/B-VI Release 8, LA-UR-03-1019, Los Alamos National Laboratory (2003). 